\title{
Factors Affecting Swedish Forest Property Prices
}

\author{
Sofia Carlsson ${ }^{1}$, Lars Lönnstedt ${ }^{2 *}$, Torbjörn Sundelin ${ }^{2}$ \\ ${ }^{1}$ Holmen Skog, Örnsköldsvik, Sweden \\ ${ }^{2}$ Dpartment of Forest Products, Swedish University of Agricultural Sciences, Uppsala, Sweden \\ Email: sofia.carlsson@holmenskog.com, ㄴars.Lonnstedt@slu.se, sundelin@slump.se
}

Received 12 May 2014; revised 14 June 2014; accepted 2 July 2014

Copyright (C) 2014 by authors and Scientific Research Publishing Inc.

This work is licensed under the Creative Commons Attribution International License (CC BY).

http://creativecommons.org/licenses/by/4.0/

(c) (i) Open Access

\begin{abstract}
Historically the price of a forest estate has, at least in Sweden, been closely related to the financial return from the estate, but this connection no longer seems to be that strong, other factors that previously had limited influence on prices now add to the price. Factors like site productivity and age distribution affect the financial return of a forest estate but is it obvious that these factors still influence prices as they once did? Over the last 20 years, the price of forest estates has increased dramatically. If development of prices has led to a weakened connection between return and price, could this be explained by the fact that previously price affecting factors have less weight when valuing forest estates? The purpose of the study was therefore to investigate how a number of factors influence the forest estate price; including site productivity and age distribution. The results shows that neither site productivity, nor age distribution have a significant impact on the forest estate price. What mainly influence the prices seems to be the standing forest volume on the estate and region. The development of the prices seems not only to have influenced the relationship between the return from the forest and the prices, but also minimized the influence of factors like age distribution and site productivity. This means that estates that are bought within the same region, at the same price per cubic meter, can have considerably different potential to gain financial return.
\end{abstract}

\section{Keywords}

Bidding, Faustman, Forest Estate, Market Value, Net Present Value, Standing Volume, Value, Winner's Curse

\section{Introduction}

During the 2000s, the price of the Swedish forest estates more than doubled (LRF Konsult). Prices have risen at *Corresponding author. 
a rate that is not consistent with the development of neither wood prices nor site quality (Skogsstatistisk årsbok, 2013). The relationship between property prices on one side and wood prices and financial return on the other hand have thereby weakened (Figure 1).

Factors such as site quality and age affect a forest estate returns. They are key variables in a net present value calculation and considered in forest valuation models such "Site Index method" (Lantmäteriet). A stand's land value is defined as the maximum return year zero. The calculation is done using Faustman's formula (Faustmann, 1849) and the result estimates the value of future timber production when costs and benefits are discounted to the year when the ground is bare. Faustman's formula assumes the selected management program will be repeated infinitely many times, and hence the calculation estimates the sum of all future revenues and expenses. Land value and value of bare forest land are two synonymous terms. Land value is here referred as $\mathrm{Bu}$, where u corresponds to the rotation time. Faustman's formula is as follows:

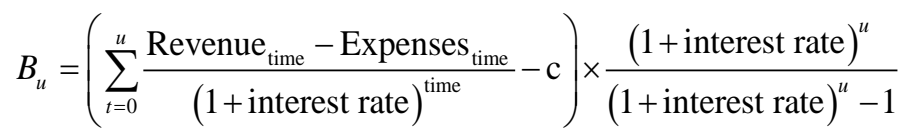

Variables including time, income, expenses and cost of capital determine the value of bare land. The variable time is dependent on site quality, which determines how far into the future various revenues and expenses will be respectively acquired and incurred. The cost of capital, in this context, is the cost of all funds required for silvicultural activities, and thus reflects the required returns on investment in a forest property, which may (for example) correspond to the interest or other monetary benefits that could be obtained from alternative investments or deposits. The property's other values, hunting rights for example, are not considered. It should be noted that there are substantial uncertainties, due to the difficulty of estimating future revenues and expenses.

The price paid for a forest estate is a product of several factors. One important factor is the buyer's valuation of the benefits (monetary and non-monetary) that the property will provide. Monetary benefits are those that can generate or be easily converted into cash, such as felling the trees, while non-monetary benefits have no readily calculable cash value, such as being able to walk freely in one's own forest. The values of both monetary and non-monetary utilities may vary substantially amongst buyers, due to differences in their perceptions of the monetary benefits of a property, weightings of the variables influencing those benefits, personal preferences and motivations (Lindeborg, 1986).

According to Thaler (1988), all bidders in an auction run the risk of a "winner's curse”, i.e. making a bad deal, because the one with the most optimistic view of an offered investment opportunity is most likely to overestimate its true value, and win (if she or he has sufficient funds). A bid that has been underpinned by valid rational

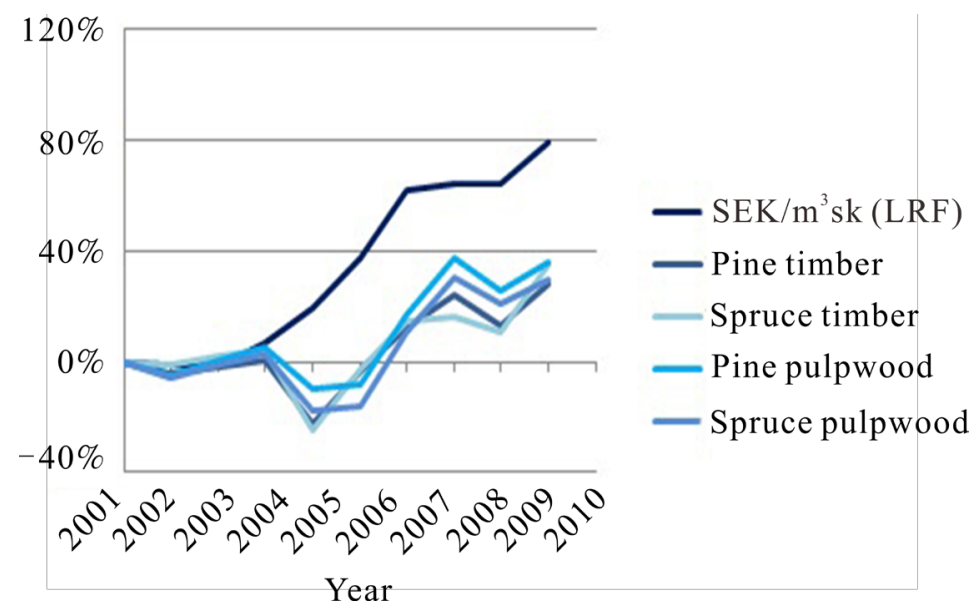

Figure 1. Changes (\%) in mean prices per unit standing volume of Swedish forests $\left(\mathrm{SEK} / \mathrm{m}^{3} \mathrm{sk}\right)$, prices for pine and spruce timber $\left(\mathrm{SEK} / \mathrm{m}^{3} \mathrm{fub}\right)$ and prices for pine and spruce pulpwood (SEK/m $\left.\mathrm{m}^{3} \mathrm{fub}\right)$. Reference year 2000 (LRF Konsult) SEK, Sk and fub are abbreviations of Swedish kronor, standing volume, roundwood excluding bark and lumber recovery factor, respectively. 
(monetary) calculations will always be lower than one based on an overestimation of the values. Thus, there is always a risk of winner's curse if bidders act irrationally, and the more interested buyers there are, the greater the risk of bids based on overestimated values.

Roos (1996) notes in a study based on 143 sales completed in 1992 that: "Forestry-related factors are of paramount importance when the price of forest land is determined. The price paid for standing volume and site quality corresponds well with the expected effect that these parameters have on the present value of future forest management. The hedonic price function can be a useful tool for examining the market for woodland and prices of forest properties.” The cited author found that the price per hectare of a forest estate was positively correlated with the share of productive forest area of the total area, the standing volume, productivity, and population density in relation to forest cover in the area, but negatively correlated with the estate's total area. However, several other authors have also explored effects of various factors using hedonic price models (Turner et al., 1991; Scarpa et al., 2000; Kennedy, 2002; Snyder et al., 2007) and the results have not been entirely consistent. In accordance with Roos (1996), some have found a negative correlation between the size and price per hectare of forest estates (for example Kennedy, 2002), but Turner et al. (1991) found no correlation between these variables. Kennedy (2002) also found support for Roos' conclusion that prices are correlated with site productivity. In addition, there are reported indications that prices are significantly negatively affected by taxes and positively related to both proximity to facilities such as ski resorts (Turner et al., 1991) and the land's general recreational value (Snyder et al., 2007). Proximity to urban areas also has a positive impact on the total price, according to Snyder et al. (2007) but a negative impact on prices per hectare according to Kennedy (2002).

Estimated supply and demand functions had also been included in some analyses, notably by Aronsson and Carlén (1997), who detected positive correlations between price and both standing volume and average site productivity, as well as a negative correlation between estate size and price per hectare, in accordance with one or both of Roos (1996) and Kennedy (2002). Their modeling also suggested that the income of the seller and purchaser had positive and negative effects on the price, respectively.

Since the study by Roos, conditions in the Swedish property market have changed for various reasons, including amendments to the Land Acquisition Act in 1991. The former purposes of the legislation were to protect family farms, safeguard employment opportunities in rural areas and limit juridical persons' opportunities to acquire land (Sveriges riksdag, 1990). Among other measures, the amendments introduced so-called "price tests" designed to ensure that prices in real estate transactions did not exceed the land's long-term returns (Lantmäteriet och Mäklarsamfundet, 2010). The law was intended to simplify and streamline the market, establishing in practice a free market in agricultural and forest land. In addition, the changes allowed people to acquire such land who had been previously excluded (Sveriges riksdag, 1990). Since the amendments the market has evolved in many respects, not least in prices. In real terms, the average price of forest estates, per cubic meter of standing volume, doubled between 2001 and 2011. However, it should be noted that mean prices of forest land in these terms was substantially higher in southern Sweden than in northern Sweden during this period.

In summary, the changes in property prices have clearly weakened the link between yield and price, which raises a number of questions regarding whether there have been shifts in buyers' views of factors influencing Swedish forest estate values, particularly site quality. Thus, the purpose of this article is to examine effects of potentially significant factors on their prices, including (inter alia) site quality, the volume of standing timber on the property, the number of stands on the property, stand ages, forest land area and region (using the five regions defined and applied by LRF Konsult. Figure 1 shows the location of the five regions. Region I consists of Eastern Götaland and Svealand, Region II of Western Götaland and Svealand, Region III of Western Svealand and Southern Norrland, Region IV of the eastern part of Northern Norrland and Region V of Western Norrland (Figure 2).

Most of the formatting specifications needed for preparing electronic versions of their papers. All standard paper components have been specified for three reasons: 1) ease of use when formatting individual papers, 2) automatic compliance to electronic requirements that facilitate the concurrent or later production of electronic products, and 3) conformity of style throughout a journal paper. Margins, column widths, line spacing, and type styles are built-in; examples of the type styles are provided throughout this document and are identified in italic type, within parentheses, following the example. Some components, such as multi-leveled equations, graphics, and tables are not prescribed, although the various table text styles are provided. The formatter will need to create these components, incorporating the applicable criteria that follow. 


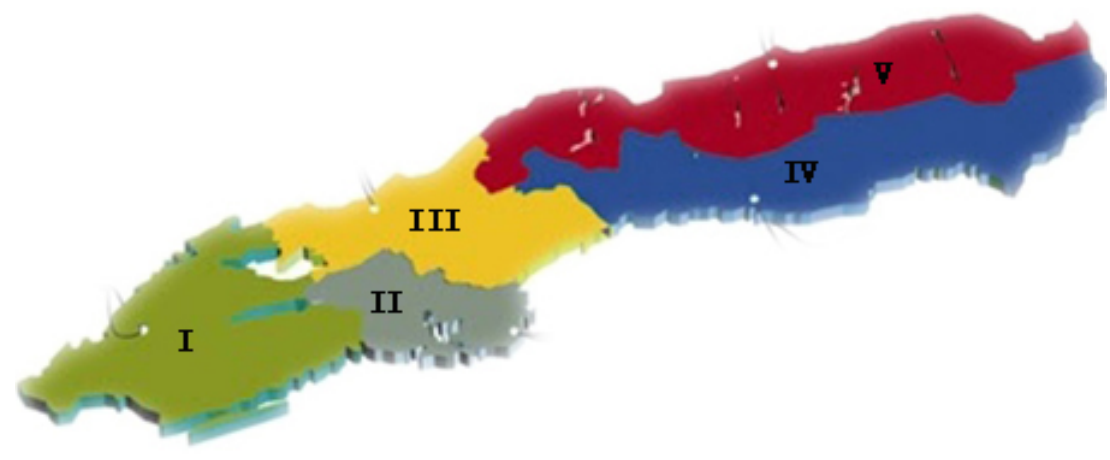

Figure 2. Five different regions in Sweden used in the analysis (LRF Konsult).

\section{Material and Method}

The dataset the study is based upon includes data for 395 properties with tax assessment values based solely or only partly on forest land, referred to hereafter as forest estates and combined estates, respectively. The data were drawn from a database compiled in efforts to develop a new tool for econometric assessments of Swedish forestry, from information on forest properties posted for sale through brokers since 2010, supplemented with sale prices from the Swedish National Land Survey. The material considered here includes only properties that were sold through brokers up until March 2012. Properties that were transferred via property border adjustments are not considered. The data for solely forest estates, combined estates and all estates were separately analyzed. The analysis based on all estates was expected to have low reliability, because variation in the other values of combined estates would confound interpretation of effects of considered factors on the price of the forest land. However, this analysis was included because increasing the number of analyzed estates could potentially help to explain changes in forest estates' prices.

The variables included in the dataset are listed and briefly described in Table 1. The variable "Age classes" listed in the table is a numerical value, calculated from area-weighted percentages of forests assigned to different age classes on the estates. The original data retrieved from the database were supplemented with a number of calculated variables (Table 2). The relationships between the price and all the studied variables are assumed to be linear.

Data were processed using Excel and Minitab software. The original dataset was transferred into two Excel spreadsheets, one with information on area per age group and one with all data excluding area per age class. The data were subsequently summarized in a single Excel sheet, values of defined variables were calculated and the dataset was transferred to Minitab for non-response analysis, and the identification of duplicates and outliers (which were removed), followed by calculation of summary statistics and stepwise regression analyses, using the remaining data, of the independent variables' effects on the listed property prices. As mentioned the relationships between these variables were assumed to be linear and thus were modelled using the following equation:

where $\alpha=$ the input value;

$$
\mathrm{y}=\alpha+\beta 1 \mathrm{x} 1+\cdots+\beta \mathrm{nxn}+\varepsilon
$$

$\beta 1=$ constant of the first included variable;

$\mathrm{x} 1=$ value of the first included variable;

$\beta \mathrm{n}=$ constant of variable $\mathrm{n}$;

$\mathrm{xn}=$ value of variable $\mathrm{n}$;

$\varepsilon=$ normally distributed random variable.

In the stepwise regression analysis variables were successively added to the model, and those that had no significant impact $(p<0.10)$ on the price were eliminated. A dummy variable was used for regions.

The goal of the statistical analysis was to identify variables that influenced the price of productive forest land in Sweden during the study period. Thus, properties with prices that were strongly influenced by other factors (e.g. properties classified as woodland, but used for industrial purposes) had to be excluded before the analysis. Such properties were identified as outliers in scatter plots (Andersson et al., 2007) of price per hectare versus forest volume per hectare, assuming that values of properties with significantly deviating prices probably included 
Table 1. Variables in the dataset.

\begin{tabular}{ll}
\hline Variable & Description \\
\hline County & Numerically designated \\
Municipality & Numerically designated \\
Parish & Numerically designated \\
Act & Estate identification number. Numerically designated \\
No. stands & Number of stands on the property \\
Ave. yield & Average site quality for the estate, $\mathrm{m}^{3}$ sk/ha/year \\
Sales pr. & Sale price of the estate, SEK \\
S:m oth (land, buildings) & Ratable value for other land than forest land and buildings, SEK \\
S:m oth (excl. Land, buildings) & Ratable value excluding land and buildings, SEK \\
Bare land & Bare land, hectare \\
Waste land & Waste land, hectare \\
Field area & Field area, hectare \\
Prod. area & Productive forest land, hectare \\
Purchase date & Purchase date of the estate \\
Standing volume & Total standing volume on the estate, $\mathrm{m}^{3}$ sk \\
Age classes & Age classes identified through a number, 1 - 15 \\
Age area & Forest area belonging to a given age class \\
\hline
\end{tabular}

Table 2. Explanation of calculated variables.

\begin{tabular}{ll}
\hline \multicolumn{1}{c}{ Variable } & \multicolumn{1}{c}{ Calculation } \\
\hline $\begin{array}{l}\text { Total forest } \\
\text { SEK } / \mathrm{m}^{3} \\
\mathrm{SEK} / \mathrm{ha}\end{array}$ & $\begin{array}{l}\text { Purchase price minus value of other land and buildings } \\
\text { Total forest value/standing volume }\end{array}$ \\
$\mathrm{m}^{3} / \mathrm{ha}$ & Total forest value/productive area \\
$>20$ year & Standing volume/productive area \\
$>40$ year & Total area in age classes $>20$ years/productive area \\
$>60$ year & Total area in age classes $>40$ years/productive area \\
Reg & Total area in age classes $>60$ years/productive area \\
Quota remaining area & Region according to LRF Konsult's classification \\
\hline
\end{tabular}

values of other, non-forest assets. Similarly, values of properties with prices significantly deviating from those of other properties in the same region probably included values of other assets. These properties were examined more closely. This resulted in the exclusion of four properties due to duplication, strong influences of other land uses or incorrect specifications of value. The calculated price per hectare of forest land for some combined agricultural estates was negative, illustrating the importance of excluding such properties. In several cases the tax assessment value of buildings and other land exceeded the sales price for the entire property. To minimize the impact of strongly deviant values the dataset was cleared as follows.

For all properties in the dataset the following ratio was calculated:

$$
\text { Proportional value of other assets }=\frac{\text { Other values (land, buidlings) }}{\text { Sales price }}
$$

All properties for which this ratio exceeded 0.5, i.e. all those for which the value of other assets accounted for at least half of the sale price, were eliminated, thus clearing the material of negative forest prices and increasing the likelihood that the remaining properties had been largely valued according to their forest resources. All 
purely forest properties in the material were retained following this procedure. In total seven properties were excluded, three due to negative forest prices and four due to duplication. In the analysis of forest estates 170 properties were included, and in the analysis of all estates 352 properties were included.

\section{Results}

\subsection{Forest Estates}

Means and standard deviations, together with minimum, median and maximum values for each of the considered variables of forest estates are shown in Table 3.

Table 3. Summary statistics for forest estates.

\begin{tabular}{|c|c|c|c|c|c|c|c|}
\hline Variable & Region & Number & Average & Standard Dev. & Minimum & Median & Maximum \\
\hline \multirow[t]{6}{*}{ SEK/ha } & 1 & 27 & 83,905 & 35,252 & 38,035 & 75,616 & 159,091 \\
\hline & 2 & 7 & 90,182 & 28,799 & 51,316 & 87,755 & 128,185 \\
\hline & 3 & 74 & 47,592 & 24,924 & 15,000 & 41,863 & 144,444 \\
\hline & 4 & 42 & 31,532 & 16,302 & 7288 & 28,783 & 82,083 \\
\hline & 5 & 20 & 27,468 & 19,345 & 9096 & 23,580 & 99,315 \\
\hline & All & 170 & 48,778 & 31,495 & 7288 & 41,386 & 159,091 \\
\hline \multirow[t]{6}{*}{ Ave yield } & 1 & 27 & 7.115 & 1.525 & 3.900 & 7.400 & 9.600 \\
\hline & 2 & 7 & 6.771 & 1.083 & 5.300 & 6.700 & 8.300 \\
\hline & 3 & 74 & 5.424 & 1.210 & 2.600 & 5.300 & 8.100 \\
\hline & 4 & 42 & 3.736 & 0.763 & 2.600 & 3.650 & 5.500 \\
\hline & 5 & 20 & 3.365 & 0.813 & 2.400 & 3.200 & 4.800 \\
\hline & All & 170 & 5.089 & 1.696 & 2.400 & 5.000 & 9.600 \\
\hline \multirow[t]{6}{*}{$\mathrm{m}^{3} / \mathrm{ha}$} & 1 & 27 & 130.06 & 39.19 & 58.67 & 130.59 & 197.03 \\
\hline & 2 & 7 & 142.7 & 29.0 & 84.1 & 148.3 & 173.8 \\
\hline & 3 & 74 & 115.52 & 50.26 & 17.01 & 111.37 & 234.05 \\
\hline & 4 & 42 & 93.15 & 41.73 & 4.95 & 88.62 & 187.57 \\
\hline & 5 & 20 & 79.30 & 36.79 & 25.49 & 75.40 & 199.45 \\
\hline & All & 170 & 109.16 & 47.28 & 4.95 & 105.96 & 234.05 \\
\hline \multirow[t]{6}{*}{$\%>20$ year } & 1 & 27 & 0.6905 & 0.1900 & 0.2919 & 0.7347 & 1.0000 \\
\hline & 2 & 7 & 0.7718 & 0.2080 & 0.3158 & 0.8545 & 0.9010 \\
\hline & 3 & 74 & 0.7439 & 0.2238 & 0.0000 & 0.7793 & 1.0000 \\
\hline & 4 & 42 & 0.7216 & 0.2323 & 0.0000 & 0.7541 & 1.0000 \\
\hline & 5 & 20 & 0.6755 & 0.2597 & 0.0424 & 0.7100 & 1.000 \\
\hline & All & 170 & 0.7230 & 0.2239 & 0.0000 & 0.7542 & 1.0000 \\
\hline \multirow[t]{6}{*}{$\%>40$ year } & 1 & 27 & 0.4707 & 0.1616 & 0.1886 & 0.4789 & 0.7778 \\
\hline & 2 & 7 & 0.5558 & 0.1854 & 0.3158 & 0.5050 & 0.8471 \\
\hline & 3 & 74 & 0.4715 & 0.2663 & 0.0000 & 0.4234 & 1.0000 \\
\hline & 4 & 42 & 0.5859 & 0.2364 & 0.0000 & 0.6248 & 1.0000 \\
\hline & 5 & 20 & 0.5064 & 0.2856 & 0.0424 & 0.4887 & 1.0000 \\
\hline & All & 170 & 0.5072 & 0.2470 & 0.0000 & 0.5101 & 1.0000 \\
\hline$\%>60$ year & 1 & 27 & 0.3337 & 0.1515 & 0.1159 & 0.3007 & 0.7778 \\
\hline
\end{tabular}




\begin{tabular}{|c|c|c|c|c|c|c|c|}
\hline \multicolumn{8}{|l|}{ Continued } \\
\hline & 2 & 7 & 0.315 & 0.279 & 0.000 & 0.273 & 0.799 \\
\hline & 3 & 74 & 0.2677 & 0.2540 & 0.0000 & 0.1994 & 1.0000 \\
\hline & 4 & 42 & 0.4615 & 0.2503 & 0.0000 & 0.4846 & 1.0000 \\
\hline & 5 & 20 & 0.3733 & 0.2719 & 0.0000 & 0.2803 & 0.9726 \\
\hline & All & 170 & 0.3404 & 0.2526 & 0.0000 & 0.2850 & 1.0000 \\
\hline \multirow[t]{6}{*}{ No. stands } & 1 & 27 & 1.259 & 0.594 & 1.000 & 1.000 & 3.000 \\
\hline & 2 & 7 & 1.0000 & 0.000000 & 1.0000 & 1.0000 & 1.0000 \\
\hline & 3 & 74 & 1.757 & 1.225 & 1.000 & 1.000 & 7.000 \\
\hline & 4 & 42 & 1.786 & 1.071 & 1.000 & 1.000 & 5.000 \\
\hline & 5 & 20 & 1.800 & 1.056 & 1.000 & 1.500 & 5.000 \\
\hline & All & 170 & 1.6588 & 1.0775 & 1.0000 & 1.0000 & 7.0000 \\
\hline \multirow[t]{6}{*}{ Prod. area } & 1 & 27 & 49.10 & 42.05 & 6.30 & 34.80 & 165.90 \\
\hline & 2 & 7 & 46.0 & 29.2 & 7.6 & 48.1 & 98.0 \\
\hline & 3 & 74 & 33.24 & 29.47 & 2.70 & 24.70 & 136.90 \\
\hline & 4 & 42 & 52.14 & 40.26 & 7.20 & 46.80 & 224.60 \\
\hline & 5 & 20 & 69.6 & 57.2 & 6.0 & 48.6 & 186.90 \\
\hline & All & 170 & 45.23 & 39.82 & 2.70 & 34.70 & 224.60 \\
\hline
\end{tabular}

The results show that the number of properties sold was highest in Region III and few were sold in Region II. The averages of the variables vary between the regions, but the differences between the mean values of all variables between Regions I and II are very small. Thus, data for Regions I and II were combined in the regression analysis, i.e. dummy variables were created for Regions III, IV and V.

Ten input variables were used in the stepwise regression. Variables for age classes showed no relation with price, and thus were not included in any of the models. Average site quality class was significantly correlated with price in Models 2 to 5, but was excluded as an explanatory variable when the Region variables were used in the analysis. The model with the highest explanatory value and the highest number of explanatory variables at the highest level of significance included six variables. The results of the stepwise regression are summarized in Table 4.

The results indicate that prices are strongly and positively correlated with volume per hectare $(p<0.01$ in all models), but negatively correlated with numbers of stands on a property and forest area. However, average site quality class was excluded when regional variables were added to the models, presumably partly because estates located in the same region have several similar attributes, including production capacity. Furthermore, no effect of site quality was detected, indicating that buyers did not take into account variations in site quality within regions. Proportional areas of stands exceeding defined ages also had no significant effects on prices, indicating that similar forest volumes in different age classes are given similar values (thus, age class variables were excluded from all models). Nevertheless, mature forests typically have larger standing volumes per hectare than younger forest, so the age distribution had indirect effects on prices through the increases per unit volume. Thus, if mature volumes had been given higher values than non-harvestable volumes, it is likely that the age class variables would have shown significant correlations with price.

As shown in Table 5, many of the variables were significantly correlated, notably average site quality class was correlated with standing volume per hectare, the proportions of forest area over 40 and 60 years old, and regions ( $p<0.05$ in each case). The correlations between average site quality class and both volume per hectare and region are consistent with expectations as fertile sites and southern sites are generally more productive than infertile and northern sites, respectively (The Swedish National Forest Inventory). A less significant correlation $(p<0.1)$ between average site quality class and productive area was also detected, partly because of variations in properties' average sizes amongst regions (which are highest in Region V and lowest in Region III). 
Table 4. Results of stepwise regression analyses of the influence of indicated variables on prices of forest estates. Reg III, Reg IV and Reg 5 are dummy variables for the influence of the data for these regions.

\begin{tabular}{|c|c|c|c|c|c|c|c|c|}
\hline Model & 1 & 2 & 3 & 4 & 5 & 6 & 7 & 8 \\
\hline$\alpha$ & -2101 & $-23,940$ & $-13,848$ & $-13,454$ & -1996 & 29,739 & 39,708 & 44,724 \\
\hline $\mathrm{m}^{3} / \mathrm{ha}$ & 466 & 375 & 368 & 374 & 376 & 363 & 372 & 366 \\
\hline$p$-value & 0.000 & 0.000 & 0.000 & 0.000 & 0.000 & 0.000 & 0.000 & 0.000 \\
\hline Ave. yield & & 6239 & 5981 & 6421 & 5108 & 1596 & & \\
\hline$p$-value & & 0.000 & 0.000 & 0.000 & 0.000 & 0.207 & & \\
\hline No. stands & & & -4781 & -4326 & -3992 & -3278 & -3218 & -2848 \\
\hline$p$-value & & & 0.001 & 0.002 & 0.004 & 0.013 & 0.015 & 0.029 \\
\hline Reg III & & & & -9375 & $-14,575$ & $-26,990$ & $-29,452$ & $-31,231$ \\
\hline$p$-value & & & & 0.003 & 0.000 & 0.000 & 0.000 & 0.000 \\
\hline Reg IV & & & & & $-13,476$ & $-32,137$ & $-37,094$ & $-37,178$ \\
\hline$p$-value & & & & & 0.002 & 0.000 & 0.000 & 0.000 \\
\hline Reg V & & & & & & $-30,535$ & $-35,959$ & $-34,434$ \\
\hline$p$-value & & & & & & 0.000 & 0.000 & 0.000 \\
\hline Prodarea & & & & & & & & -97 \\
\hline$p$-value & & & & & & & & 0.008 \\
\hline $\mathrm{R}^{2}$ (adj) & 48.65 & 57.89 & 60.31 & 62.22 & 64.11 & 67.81 & 67.69 & 68.88 \\
\hline
\end{tabular}

Table 5. Correlation matrix for forest estates.

\begin{tabular}{|c|c|c|c|c|c|c|c|c|c|}
\hline & Ave. yield & $\mathrm{m}^{3} / \mathrm{ha}$ & $\%>20$ year & $\%>40$ year & $\%>60$ year & Reg III & $\begin{array}{c}\text { Reg } \\
\text { IV }\end{array}$ & Reg V & $\begin{array}{l}\text { No. } \\
\text { stands }\end{array}$ \\
\hline $\mathrm{m}^{3} / \mathrm{ha}$ & $\begin{array}{l}0.406 \\
0.000\end{array}$ & & & & & & & & \\
\hline$\%>20$ year & $\begin{array}{l}0.009 \\
0.907\end{array}$ & $\begin{array}{l}0.579 \\
0.000\end{array}$ & & & & & & & \\
\hline$\%>40$ year & $\begin{array}{c}-0.137 \\
0.076\end{array}$ & $\begin{array}{l}0.596 \\
0.000\end{array}$ & $\begin{array}{l}0.662 \\
0.000\end{array}$ & & & & & & \\
\hline$\%>60$ year & $\begin{array}{c}-0.274 \\
0.000\end{array}$ & $\begin{array}{l}0.499 \\
0.000\end{array}$ & $\begin{array}{l}0.400 \\
0.000\end{array}$ & $\begin{array}{l}0.683 \\
0.000\end{array}$ & & & & & \\
\hline Reg III & $\begin{array}{l}0.174 \\
0.023\end{array}$ & $\begin{array}{l}0.118 \\
0.124\end{array}$ & $\begin{array}{l}0.082 \\
0.287\end{array}$ & $\begin{array}{c}-0.127 \\
0.098\end{array}$ & $\begin{array}{c}-0.254 \\
0.001\end{array}$ & & & & \\
\hline Reg IV & $\begin{array}{c}-0.458 \\
0.000\end{array}$ & $\begin{array}{c}-0.195 \\
0.011\end{array}$ & $\begin{array}{c}-0.004 \\
0.963\end{array}$ & $\begin{array}{l}0.183 \\
0.017\end{array}$ & $\begin{array}{l}0.275 \\
0.000\end{array}$ & $\begin{array}{c}-0.503 \\
0.000\end{array}$ & & & \\
\hline Reg V & $\begin{array}{c}-0.372 \\
0.000\end{array}$ & $\begin{array}{c}-0.231 \\
0.002\end{array}$ & $\begin{array}{c}-0.078 \\
0.314\end{array}$ & $\begin{array}{c}-0.001 \\
0.987\end{array}$ & $\begin{array}{l}0.048 \\
0.537\end{array}$ & $\begin{array}{c}-0.321 \\
0.000\end{array}$ & $\begin{array}{c}-0.209 \\
0.006\end{array}$ & & \\
\hline No. stands & $\begin{array}{c}-0.114 \\
0.139\end{array}$ & $\begin{array}{c}-0.106 \\
0.169\end{array}$ & $\begin{array}{c}-0.009 \\
0.911\end{array}$ & $\begin{array}{c}-0.004 \\
0.958\end{array}$ & $\begin{array}{l}0.028 \\
0.716\end{array}$ & $\begin{array}{l}0.080 \\
0.300\end{array}$ & $\begin{array}{l}0.068 \\
0.381\end{array}$ & $\begin{array}{l}0.048 \\
0.534\end{array}$ & \\
\hline Prod. area & $\begin{array}{c}-0.150 \\
0.051\end{array}$ & $\begin{array}{c}-0.136 \\
0.077\end{array}$ & $\begin{array}{c}-0.039 \\
0.612\end{array}$ & $\begin{array}{c}-0.125 \\
0.105\end{array}$ & $\begin{array}{l}0.024 \\
0.759\end{array}$ & $\begin{array}{c}-0.265 \\
0.000\end{array}$ & $\begin{array}{l}0.100 \\
0.196\end{array}$ & $\begin{array}{l}0.224 \\
0.003\end{array}$ & $\begin{array}{l}0.098 \\
0.204\end{array}$ \\
\hline
\end{tabular}

Note: Explanation: correlation; $p$-value.

\subsection{All Estates}

Means, standard deviations as well as minimum, median and maximum values for each of the variables of all estates are shown in Table 6.

The results show that the highest numbers of properties were sold in Region III and the lowest in Region II, as found in the previous analysis. The regional patterns of average values of the variables were also very similar. Inter alia, differences between mean values for Regions 1 and 2 were still very small so dummy variables were created for Regions I + II, III, IV and V. 
Table 6. Summary statistics for the total material.

\begin{tabular}{|c|c|c|c|c|c|c|c|}
\hline Variable & Region & Number & Average & Standard Deviation & Minimum & Median & Maximum \\
\hline \multirow[t]{6}{*}{ SEK/ha } & 1 & 93 & 84,292 & 37,021 & 23,208 & 77,161 & 219,928 \\
\hline & 2 & 20 & 86,834 & 43,435 & 22,165 & 81,819 & 208,704 \\
\hline & 3 & 103 & 49,516 & 28,921 & 15,000 & 42,512 & 207,978 \\
\hline & 4 & 87 & 34,114 & 20,072 & 7288 & 29,590 & 139,246 \\
\hline & 5 & 49 & 29,629 & 18,398 & 9096 & 24,273 & 99,315 \\
\hline & All & 352 & 54,249 & 36,616 & 7288 & 45,340 & 219,928 \\
\hline \multirow[t]{6}{*}{ Ave. yield } & 1 & 93 & 7.340 & 1.416 & 3.900 & 7.400 & 10.600 \\
\hline & 2 & 20 & 6.870 & 0.907 & 5.300 & 6.850 & 8.700 \\
\hline & 3 & 103 & 5.426 & 1.215 & 2.600 & 5.300 & 8.600 \\
\hline & 4 & 87 & 3.9575 & 0.7653 & 2.6000 & 3.9000 & 5.7000 \\
\hline & 5 & 49 & 3.478 & 0.727 & 2.400 & 3.500 & 5.100 \\
\hline & All & 352 & 5.3795 & 1.8349 & 2.4000 & 5.1000 & 10.6000 \\
\hline \multirow[t]{6}{*}{$\mathrm{m}^{3} / \mathrm{ha}$} & 1 & 93 & 138.58 & 42.97 & 37.76 & 130.59 & 266.39 \\
\hline & 2 & 20 & 140.89 & 40.32 & 79.52 & 136.03 & 248.18 \\
\hline & 3 & 103 & 116.43 & 49.85 & 17.01 & 110.26 & 266.50 \\
\hline & 4 & 87 & 98.98 & 43.44 & 4.95 & 93.92 & 226.74 \\
\hline & 5 & 49 & 83.95 & 40.51 & 18.82 & 77.65 & 199.45 \\
\hline & All & 352 & 114.84 & 48.63 & 4.95 & 110.28 & 266.50 \\
\hline \multirow[t]{6}{*}{$\%>20$ year } & 1 & 93 & 0.7388 & 0.1659 & 0.2919 & 0.7541 & 1.0000 \\
\hline & 2 & 20 & 0.7586 & 0.1625 & 0.3158 & 0.7782 & 1.0000 \\
\hline & 3 & 103 & 0.7569 & 0.2069 & 0.0000 & 0.8072 & 1.0000 \\
\hline & 4 & 87 & 0.7346 & 0.2183 & 0.0000 & 0.7772 & 1.0000 \\
\hline & 5 & 49 & 0.7374 & 0.2218 & 0.0424 & 0.7467 & 1.0000 \\
\hline & All & 352 & 0.7440 & 0.1990 & 0.0000 & 0.7712 & 1.0000 \\
\hline \multirow[t]{6}{*}{$\%>40$ year } & 1 & 93 & 0.5270 & 0.1800 & 0.1000 & 0.5155 & 0.9550 \\
\hline & 2 & 20 & 0.5400 & 0.1823 & 0.2390 & 0.5267 & 0.9091 \\
\hline & 3 & 103 & 0.4770 & 0.2494 & 0.0000 & 0.4490 & 1.0000 \\
\hline & 4 & 87 & 0.5670 & 0.2148 & 0.0000 & 0.5745 & 1.0000 \\
\hline & 5 & 49 & 0.5484 & 0.2548 & 0.0424 & 0.5586 & 1.0000 \\
\hline & All & 352 & 0.5260 & 0.2230 & 0.0000 & 0.5334 & 1.0000 \\
\hline \multirow[t]{6}{*}{$\%>60$ year } & 1 & 93 & 0.3499 & 0.1912 & 0.0194 & 0.3094 & 0.9028 \\
\hline & 2 & 20 & 0.3539 & 0.2430 & 0.0000 & 0.3276 & 0.7994 \\
\hline & 3 & 103 & 0.2691 & 0.2398 & 0.0000 & 0.2229 & 1.0000 \\
\hline & 4 & 87 & 0.4228 & 0.2236 & 0.0000 & 0.4218 & 1.0000 \\
\hline & 5 & 49 & 0.4076 & 0.2525 & 0.0000 & 0.3622 & 0.9787 \\
\hline & All & 352 & 0.3525 & 0.2327 & 0.0000 & 0.3188 & 1.0000 \\
\hline \multirow[t]{6}{*}{ No. stands } & 1 & 93 & 1.4731 & 0.8672 & 1.0000 & 1.0000 & 6.0000 \\
\hline & 2 & 20 & 1.550 & 1.050 & 1.000 & 1.000 & 5.000 \\
\hline & 3 & 103 & 1.816 & 1.243 & 1.000 & 1.000 & 7.000 \\
\hline & 4 & 87 & 2.195 & 1.690 & 1.000 & 2.000 & 11.000 \\
\hline & 5 & 49 & 2.000 & 1.099 & 1.000 & 2.000 & 5.000 \\
\hline & All & 352 & 1.8295 & 1.2830 & 1.0000 & 1.0000 & 11.0000 \\
\hline \multirow[t]{6}{*}{ Prod. areal } & 1 & 93 & 44.53 & 43.79 & 6.30 & 33.60 & 298.30 \\
\hline & 2 & 20 & 50.8 & 48.4 & 7.6 & 31.4 & 209.90 \\
\hline & 3 & 103 & 33.67 & 30.96 & 2.70 & 22.90 & 147.20 \\
\hline & 4 & 87 & 52.94 & 46.04 & 4.20 & 41.50 & 236.60 \\
\hline & 5 & 49 & 66.66 & 57.87 & 2.50 & 48.20 & 291.70 \\
\hline & All & 352 & 46.87 & 44.75 & 2.50 & 33.45 & 298.30 \\
\hline
\end{tabular}


The properties that were added to this analysis of the whole material, i.e. properties whose total market value reflects values of other assets in addition to woodland were also analyzed separately (Table 7).

Table 7. Summary statistics for combined estates.

\begin{tabular}{|c|c|c|c|c|c|c|c|}
\hline Variable & Region & Number & Averge & Standard Deviation & Minimum & Median & Maximum \\
\hline \multirow[t]{6}{*}{$\mathrm{kr} / \mathrm{ha}$} & 1 & 65 & 82,514 & 34,841 & 23,208 & 77,161 & 219,928 \\
\hline & 2 & 13 & 85,031 & 50,620 & 22,165 & 63,812 & 208,704 \\
\hline & 3 & 29 & 54,425 & 37,317 & 16,266 & 46,867 & 207,978 \\
\hline & 4 & 45 & 36,524 & 22,967 & 9238 & 30,099 & 139,246 \\
\hline & 5 & 29 & 31,119 & 17,908 & 9493 & 24,421 & 66,401 \\
\hline & All & 181 & 58,526 & 38,745 & 9238 & 52,977 & 219,928 \\
\hline \multirow[t]{6}{*}{ Ave. yield } & 1 & 65 & 7.445 & 1.378 & 4.000 & 7.400 & 10.600 \\
\hline & 2 & 13 & 6.923 & 0.841 & 5.800 & 7.000 & 8.700 \\
\hline & 3 & 29 & 5.431 & 1.250 & 2.900 & 5.200 & 8.600 \\
\hline & 4 & 45 & 4.164 & 0.715 & 2.600 & 4.100 & 5.700 \\
\hline & 5 & 29 & 3.555 & 0.666 & 2.600 & 3.500 & 5.100 \\
\hline & All & 181 & 5.646 & 1.925 & 2.600 & 5.400 & 10.600 \\
\hline \multirow[t]{6}{*}{$\mathrm{m}^{3} / \mathrm{ha}$} & 1 & 65 & 142.73 & 44.24 & 37.76 & 130.77 & 266.39 \\
\hline & 2 & 13 & 139.9 & 46.4 & 79.5 & 126.6 & 248.20 \\
\hline & 3 & 29 & 118.74 & 49.58 & 46.63 & 110.26 & 266.50 \\
\hline & 4 & 45 & 104.42 & 44.75 & 40.40 & 95.01 & 226.74 \\
\hline & 5 & 29 & 87.16 & 43.22 & 18.82 & 77.65 & 199.39 \\
\hline & All & 181 & 120.26 & 49.51 & 18.82 & 113.94 & 266.50 \\
\hline \multirow[t]{6}{*}{$\%>20$ year } & 1 & 65 & 0.7581 & 0.1532 & 0.4636 & 0.7681 & 1.0000 \\
\hline & 2 & 13 & 0.7516 & 0.1416 & 0.5022 & 0.7654 & 1.0000 \\
\hline & 3 & 29 & 0.7900 & 0.1545 & 0.4743 & 0.8111 & 1.0000 \\
\hline & 4 & 45 & 0.7467 & 0.2062 & 0.1970 & 0.7791 & 1.0000 \\
\hline & 5 & 29 & 0.7800 & 0.1843 & 0.3348 & 0.8117 & 1.0000 \\
\hline & All & 181 & 0.7635 & 0.1712 & 0.1970 & 0.7875 & 1.0000 \\
\hline \multirow[t]{6}{*}{$\%>40$ year } & 1 & 65 & 0.5489 & 0.1843 & 0.1000 & 0.5418 & 0.9550 \\
\hline & 2 & 13 & 0.5315 & 0.1877 & 0.2390 & 0.5484 & 0.9091 \\
\hline & 3 & 29 & 0.4910 & 0.2032 & 0.0323 & 0.5029 & 0.7854 \\
\hline & 4 & 45 & 0.5494 & 0.1936 & 0.0606 & 0.5415 & 0.9855 \\
\hline & 5 & 29 & 0.5774 & 0.2320 & 0.1255 & 0.5766 & 1.0000 \\
\hline & All & 181 & 0.5431 & 0.1976 & 0.0323 & 0.5458 & 1.0000 \\
\hline \multirow[t]{6}{*}{$\%>60$ year } & 1 & 65 & 0.3590 & 0.2064 & 0.0194 & 0.3125 & 0.9028 \\
\hline & 2 & 13 & 0.3747 & 0.2305 & 0.0000 & 0.3786 & 0.7159 \\
\hline & 3 & 29 & 0.2728 & 0.2029 & 0.0000 & 0.2333 & 0.6758 \\
\hline & 4 & 45 & 0.3868 & 0.1913 & 0.0000 & 0.8810 & 0.3687 \\
\hline & 5 & 29 & 0.4313 & 0.2403 & 0.0631 & 0.3858 & 0.9787 \\
\hline & All & 181 & 0.3648 & 0.2127 & 0.0000 & 0.3514 & 0.9787 \\
\hline \multirow[t]{6}{*}{ No. stands } & 1 & 65 & 1.569 & 0.951 & 1.000 & 1.000 & 6.000 \\
\hline & 2 & 13 & 1.846 & 1.214 & 1.000 & 1.000 & 5.000 \\
\hline & 3 & 29 & 1.966 & 1.295 & 1.000 & 1.000 & 5.000 \\
\hline & 4 & 45 & 2.578 & 2.050 & 1.000 & 2.000 & 11.000 \\
\hline & 5 & 29 & 2.138 & 1.125 & 1.000 & 2.000 & 4.000 \\
\hline & All & 181 & 1.994 & 1.436 & 1.000 & 1.000 & 11.000 \\
\hline \multirow[t]{6}{*}{ Prod. area } & 1 & 65 & 43.13 & 44.85 & 6.90 & 31.20 & 298.30 \\
\hline & 2 & 13 & 53.4 & 57.1 & 8.8 & 28.1 & 209.90 \\
\hline & 3 & 29 & 34.76 & 35.00 & 5.30 & 20.00 & 147.20 \\
\hline & 4 & 45 & 53.69 & 51.29 & 4.20 & 38.00 & 236.60 \\
\hline & 5 & 29 & 64.6 & 59.3 & 2.5 & 46.6 & 291.70 \\
\hline & All & 181 & 48.59 & 49.03 & 2.50 & 32.30 & 298.30 \\
\hline
\end{tabular}


The results show that the properties added for the analysis of all the material had an average price of just under 10,000 SEK more per hectare, and slightly higher average site quality class and standing volume per hectare than purely forest properties. These findings are consistent with expectations because there was a significantly higher proportion of such properties than forest estates in the southern regions.

As in the previous analysis, 10 input variables were included in the stepwise regression analysis of this dataset, results of which are summarized in Table 8.

Model 7 showed a marginally significant positive correlation $(p<0.1)$ between prices and the areal proportion of forest more than 40 years old (in contrast to the absence of age-price correlations detected in the previous analysis), but the other age-related variables were not significantly correlated with prices. The model with the highest coefficient of determination, number of explanatory variables and significance level included five variables. The results differ somewhat from those of the previous analysis as average site quality class had a significant impact on the market price even when regions were added to the model in step 7.

Correlations between the variables are shown in Table 9 .

As in the previous analysis, significant correlations between several variables were detected, including $p<$ 0.01 correlations for average site quality class with standing volume per hectare, the proportion of forest over 60 years old, region, the number of stands and production area. The correlations of average site quality class with standing volume per hectare, region and production area are consistent with expectations, for reasons that have already been stated. The correlation between age and average site quality class could be due to the negative association between site quality class and rotation time, as properties with low site quality are likely to have older than average forests. Two main contributors to the regional correlations are the variations in average property size (highest in Region V and lowest in Region III) and deviations in site quality class from mean values in Region III.

\subsection{Summary of the Regression Analyses' Results}

According to both analyses the market prices of the estates per hectare were strongly correlated with standing volume per hectare, with region significantly but less strongly, and marginally significantly with their forest area. In the analysis of purely forest properties a weak but significant correlation with the number of stands was also detected. However, no significant correlation was detected between price and either site quality class or areal proportions of the forest exceeding defined ages in the analysis of purely forest properties, although weak correlations $(p<0.10)$ were found between price and both average site quality class and share of forest over 40 years old in the analysis of all properties. The only variables that contributed to explanation of the prices for all properties at $95 \%$ or $99 \%$ significance levels were standing volume per hectare, region and production area. The model had less explanatory power than the model generated from analysis of the forest estates, thus addition of data for the combined estates did not enhance the reliability of the analysis.

The results indicate that standing volume was the most important determinant of the price of Swedish forest land during the study period, but region also had a significant influence (which was not solely related to variations in site quality class between regions). The results also indicate that the number of stands and production area were probably related to the price, but not the maturity of the forest (or, more specifically, areal proportions of the forest exceeding given ages).

\section{Discussion}

The results of our analyses indicate that the price of forest land in Sweden during the study period, which is very close to current, was largely determined by two variables: the volume of standing timber per hectare, and the geographic region of the property. Neither site quality class nor age distribution of the forest seemed to significantly affect market prices, in contrast to findings of several previous analyses (Roos, 1996; Kennedy, 2002; Aronsson \& Carlén, 1997). This poses several questions regarding apparent shifts in importance of the considered factors, the degree to which the discrepancy can be explained by differences between monetary and nonmonetary values, and the relationship of the results (if any) to winner's curse theory.

It should be noted that the grouping of estates in the same geographic regions will also have resulted, to some extent, in grouping of properties with other similarities, including similarities in returns from forestry and (to some extent) demographic and infrastructural properties of their locations. The regional affiliation contributes to the explanation of the property prices, which generally increase from north to south. 
Table 8. Results of stepwise regression analyses of the influence of indicated variables on prices of all estates. Reg III, Reg IV and Reg 5 are dummy variables for the influence of the data for these regions.

\begin{tabular}{|c|c|c|c|c|c|c|c|c|}
\hline Model & 1 & 2 & 3 & 4 & 5 & 6 & 7 & 8 \\
\hline$\alpha$ & 2466.4 & -23987.7 & -16689.2 & -12588.1 & 345.2 & 32693.3 & 24,846 & 47,222 \\
\hline $\mathrm{m}^{3} / \mathrm{ha}$ & 451 & 318 & 309 & 309 & 311 & 300 & 235 & 312.99 \\
\hline$p$-value & 0.000 & 0.000 & 0.000 & 0.000 & 0.000 & 0.000 & 0.000 & 0.000 \\
\hline Ave. yield & & 7764 & 7466 & 7429 & 5946 & 2226 & 3236 & \\
\hline$p$-value & & 0.000 & 0.000 & 0.000 & 0.000 & 0.072 & 0.016 & \\
\hline prod. area & & & -100 & -120 & -129 & -126 & -119 & -131.09 \\
\hline$p$-value & & & 0.002 & 0.000 & 0.000 & 0.000 & 0.000 & 0.000 \\
\hline Reg III & & & & $-10,117$ & $-14,989$ & $-25,900$ & $-24,518$ & $-29,733$ \\
\hline$p$-value & & & & 0.001 & 0.000 & 0.000 & 0.000 & 0.000 \\
\hline Reg IV & & & & & $-13,745$ & $-30,367$ & $-30,348$ & $-37,147$ \\
\hline$p$-value & & & & & 0.000 & 0.000 & 0.000 & 0.000 \\
\hline Reg V & & & & & & $-27,545$ & $-27,799$ & $-35,130$ \\
\hline$p$-value & & & & & & 0.000 & 0.000 & 0.000 \\
\hline$\%>40$ year & & & & & & & 17,394 & \\
\hline$p$-value & & & & & & & 0.054 & \\
\hline $\mathrm{R}^{2}$ (adj) & 35.68 & 47.57 & 48.88 & 50.28 & 51.92 & 54.38 & 54.74 & 54.1 \\
\hline
\end{tabular}

Table 9. Correlation matrix for the whole material.

\begin{tabular}{|c|c|c|c|c|c|c|c|c|c|}
\hline & Ave. yield & $\mathrm{m}^{3} / \mathrm{ha}$ & $\%>20$ year & $\%>40$ year & $\%>60$ year & Reg III & Reg IV & Reg V & No. stands \\
\hline $\mathrm{m}^{3} / \mathrm{ha}$ & $\begin{array}{l}0.455 \\
0.000\end{array}$ & & & & & & & & \\
\hline$\%>20$ year & $\begin{array}{c}-0.020 \\
0.704\end{array}$ & $\begin{array}{l}0.531 \\
0.000\end{array}$ & & & & & & & \\
\hline$\%>40$ year & $\begin{array}{c}-0.103 \\
0.053\end{array}$ & $\begin{array}{l}0.602 \\
0.000\end{array}$ & $\begin{array}{l}0.632 \\
0.000\end{array}$ & & & & & & \\
\hline$\%>60$ year & $\begin{array}{c}-0.215 \\
0.000\end{array}$ & $\begin{array}{l}0.479 \\
0.000\end{array}$ & $\begin{array}{l}0.424 \\
0.000\end{array}$ & $\begin{array}{l}0.728 \\
0.000\end{array}$ & & & & & \\
\hline Reg III & $\begin{array}{l}0.016 \\
0.759\end{array}$ & $\begin{array}{l}0.021 \\
0.694\end{array}$ & $\begin{array}{l}0.042 \\
0.434\end{array}$ & $\begin{array}{c}-0.141 \\
0.008\end{array}$ & $\begin{array}{c}-0.231 \\
0.000\end{array}$ & & & & \\
\hline Reg IV & $\begin{array}{c}-0.445 \\
0.000\end{array}$ & $\begin{array}{c}-0.187 \\
0.000\end{array}$ & $\begin{array}{c}-0.027 \\
0.614\end{array}$ & $\begin{array}{l}0.106 \\
0.048\end{array}$ & $\begin{array}{l}0.173 \\
0.001\end{array}$ & $\begin{array}{c}-0.369 \\
0.000\end{array}$ & & & \\
\hline Reg V & $\begin{array}{c}-0.417 \\
0.000\end{array}$ & $\begin{array}{c}-0.256 \\
0.000\end{array}$ & $\begin{array}{c}-0.013 \\
0.803\end{array}$ & $\begin{array}{l}0.040 \\
0.449\end{array}$ & $\begin{array}{l}0.095 \\
0.074\end{array}$ & $\begin{array}{c}-0.259 \\
0.000\end{array}$ & $\begin{array}{c}-0.230 \\
0.000\end{array}$ & & \\
\hline No. stands & $\begin{array}{c}-0.182 \\
0.001\end{array}$ & $\begin{array}{c}-0.142 \\
0.008\end{array}$ & $\begin{array}{c}-0.037 \\
0.490\end{array}$ & $\begin{array}{c}-0.048 \\
0.373\end{array}$ & $\begin{array}{l}0.025 \\
0.636\end{array}$ & $\begin{array}{c}-0.007 \\
0.895\end{array}$ & $\begin{array}{l}0.164 \\
0.002\end{array}$ & $\begin{array}{l}0.054 \\
0.317\end{array}$ & \\
\hline Prod. area & $\begin{array}{c}-0.164 \\
0.002\end{array}$ & $\begin{array}{c}-0.149 \\
0.005\end{array}$ & $\begin{array}{c}-0.043 \\
0.423\end{array}$ & $\begin{array}{c}-0.096 \\
0.072\end{array}$ & $\begin{array}{c}-0.024 \\
0.648\end{array}$ & $\begin{array}{c}-0.190 \\
0.000\end{array}$ & $\begin{array}{l}0.078 \\
0.145\end{array}$ & $\begin{array}{l}0.178 \\
0.001\end{array}$ & $\begin{array}{l}0.206 \\
0.000\end{array}$ \\
\hline
\end{tabular}

Note: Correlation; $p$-value.

However, there may also be substantial variations among estates located within the same region in numerous factors that affect returns from forestry, including site quality and the age of the stands. Thus, the absence of any detected significant effect of these variables on the price indicates that they were not taken into account in the valuation of properties located within the same region. If so, the key determinants of net present value did not proportionately impact property prices, and market prices deviated from those that would result from net present value calculations. These findings clearly indicate that the buyers of forest land in Sweden during the study period did not base their valuations solely on expected financial returns. Thus, they acted to some degree at least irrationally (in monetary terms), and less rationally, apparently, than in quite recent periods covered by previous analyses (Roos, 1996; Kennedy, 2002; Snyder et al., 2007). 
The price of forest estates may have risen faster than that of associated assets (net present value) because non-monetary benefits are attributed higher values than previously (and thus valuations are still essentially rational: high monetary or non-monetary values result in high property prices). Alternatively, the apparent absence of significant effects of site quality and forest age on property prices could be due to a general lack of knowledge of properties' true monetary values. Key questions are whether buyers rationally assess properties' nonmonetary values when the size of monetary resources is partially unknown and whether they can consciously evaluate non-monetary values without first having assessed monetary values. Several factors must be considered to address these questions, particularly the difference between value and price. A property's total value for a given potential buyer is the sum of his or her estimates of the monetary and non-monetary values, hence the non-monetary value placed on a property by a buyer could theoretically be calculated simply by subtracting the monetary value (net present value) from its price. However, non-monetary values can be under or over-estimated, like all other values. For example, the value of having a seaside or lakeside location may be overestimated because a buyer can spend less time than hoped at a property, or underestimated because the fishing quality is better than anticipated, just as its financial performance may fail to meet (or exceed) expectations.

Our results do not exclude the possibility that the property prices are completely unrelated to the net present value, since it is possible that buyers do not specify and estimate the value of various non-monetary benefits before bidding for properties, although it seems more likely that they do not estimate non-monetary values rationally. Nevertheless, the regression analyses suggest that the properties' potential profitability did not affect their prices to a rational extent, and if their monetary values were not rationally estimated, it is difficult to see why their non-monetary values would be.

It should be noted that high values of non-monetary benefits may not pose risks, if they have been rationally estimated based on personal preferences, such as "Mushroom-picking is worth SEK 5000 per hectare to me". However, if the non-monetary value is unspecified or erroneous, the valuation becomes irrational and the risk of poor estimates increases. For a buyer to be able to maximize the prospects of a good deal, she or he must have reasonable understanding of all the relevant values.

A major issue that remains to be resolved (indeed an issue that in large part prompted the study) is the reason why estate prices sharply rose during the study period if the property prices did not correspond to their net present values, and changes in non-monetary values do not necessarily explain the price increase (as discussed above). The key question is whether it is valid to assume that prices have rational foundations, and winner's curse theory offers an alternative explanation that does not rely on the assumption that the valuation of estates is always based on rational analysis. For a purchaser to be at risk of winner's curse, two criteria must be met: the purchase must take place through bidding and the buyer must act irrationally, at least to some extent. The first criterion is generally fulfilled in this context because buying estates normally involves bidding, and the second criterion may be met because, for example, no relationship between price and either site quality or age distribution was detected. If the winner's curse is a common phenomenon in the estate market, prices may have changed according to the following scenario (assuming that all bidders can afford to pay a sum corresponding to their estimated value for a property).

A property is offered for sale. Potential buyers examine the property according to their requirements for information; some carefully read the real estate agent's prospectus, thoroughly study the forest plan, visit the property to form their own opinion about the state of the forest, and then assess the financial prospects using their own knowledge and experts' evaluations. Others may take a more relaxed approach or apply unpredictable cri- teria. For example, the availability of a neighboring property for the first time in 100 years may be the decisive factor for someone to place a bid. Another potential buyer may be content to study the prospectus and attempt to estimate the price based on price statistics. In some cases there may be a "bidding war", with buyers successively raising bids (particularly if there is animosity between them), until the price is finally set. Buyers who gradually withdraw from the bidding do so because bids rise above their estimated value of the property (unless they bid more than this because of some other personal motivation). The winner of the bidding process will be the person who makes the highest bid, and thus has the most optimistic estimate of estate's value (unless, again she or he has over-bid for some personal reason).

In the above example, the winning bid is (as usual) the highest. If the buyer's valuation is based on insufficient information about the property's value, and/or her/his valuation is irrational (perhaps because the price is less than the region's average, according to the statistics, or because she/he merely wanted to own the property), she or he may suffer from winner's curse. In auctions or other bidding processes a bid based on over-estimated 
value will always beat a thoroughly rational bid, because the highest bid wins. The risk of a buyer overestimating the value of a resource is greatest when knowledge about the resource is low, and if buyers generally have inadequate knowledge for rational estimates, there are increased risks of those buying forest estates suffering from winner's curse. If a buyer suffers from winner's curse she or he will either lose money or make a smaller profit than expected, because the investment has lower value than anticipated. In both situations, the buyer has invested more in an estate than the returns (monetary and non-monetary).

The regional patterns also warrant further consideration in this respect. Properties located within the same region have substantial social, ecological and economic similarities, but as shown by the statistics in Table 1 and Table 2 they are far from identical. Thus, property prices are likely to be regionally grouped to some extent, but should certainly not be homogeneous within regions. For example, Region V consists of Jämtland and Lapland counties, which differ in all listed respects (social, ecological and commercial). Thus, the price of forest properties could be expected to vary between Jämtland and Lapland, but if the price of forest land in the material had varied considerably between these counties the contribution of Region $\mathrm{V}$ would, presumably, have been weaker. A possible explanation is that LRF Konsult's price statistics may influence rather than merely reflect the market, i.e. buyers may take into account regional differences in prices, thus contributing irrationally to regions being explanatory variables in the pricing model.

The results show that the price of forest estates is influenced by region, but not site quality or age variables, possibly because some buyers (at least) simplify and generalize when valuing properties. The possibility that the price statistics may be used as a valuation instrument cannot be excluded, i.e. potential buyers may check local prices of unit volumes of wood and multiply them by the standing volumes of offered properties. If the standing volume is valued without considering the age structure of the forest, young forest will be valued using the same unit volume price as mature forest. In support of this conclusion, Skogssällskapet's consultant Ulrik Abelson (2011) reports that he discourages sellers from thinning before selling forest land, because the net price per cubic meter obtained from thinning is unlikely to exceed the estimated price of the lost standing volume at a bid.

It should be noted that the absence of expected effects on price of variables that influence the net present value does not necessarily mean that buyers make poor decisions. The present net value of forests is not the only factor that determines the financial returns from a property transaction. For example, tax advantages may be key factors for a buyer and, if so, factors such as age structure and site quality might have minor effects on profitability. Moreover, forest land may continue to increase in value, so purchases may be made for rational, speculative purposes. Nevertheless, if real estate prices are based on simplified valuation methods that do not comply with present value calculations, properties with similar yield potential may be sold at very different prices.

It is possible that properties with the same volume of standing timber, located in the same region, command similar prices per cubic meter and (thus) a naturally regenerated, uncleaned and unthinned forest may be valued as highly as an artificially regenerated, well-thinned forest with the same standing volume. If so, those who invest in the well-managed forest are likely to enjoy significantly higher returns on their investment. The same applies if forest estates with similar standing volumes but different average site indices are equally valued: investment in a property with higher site quality is likely to yield higher returns. Furthermore, properties with large standing volumes will be sold at high prices regardless of forest quality. This means that sellers prioritizing high-volume production above high present values may be able to sell their forest estates at higher prices than those managing their forests in order to maximize net present value.

\section{Conclusion}

Region and standing volume per hectare are predictive variables for the price of forest properties in our dataset. In contrast, site quality class and forest age variables (proportions of forest area over 20, 40 or 60 years old) have the most marginal effects on price, while increases in both the number of stands and area of productive forest have negative effects. These findings collectively indicate that the net present value may not have been taken into account to the expected extent if the properties had been valued on the basis of a rational economic perspectively. Furthermore, non-monetary values do not clearly explain the price rise in the property market, but winner's curse theory provides an alternative explanation for the rise.

Several limitations of our analyses that may have influenced the results should be considered. Firstly, we assumed that the relationships between forest estate prices and the tested variables were linear, but this was not necessarily true since the relationships may be curvilinear. Secondly, the efforts to exclude properties that could have biased the findings because their prices were probably influenced by values of non-forest assets based on 
deviations from patterns in scatter plots. An alternative would have been to examine characteristics of every sold property, i.e. screen outliers more rigorously, which would probably have improved the results. Thirdly, the analyzed material was essentially drawn from prospectuses describing the offered properties, which might have included incorrect details regarding (for instance) standing volumes, species distributions and site quality. However, the buyers' valuations of the properties were probably based on the same data. Thus, possible inaccuracies in the properties' descriptions did not necessarily invalidate the conclusions about the considered variables' effects on price.

A strength of the study is that the analyzed material is very close to current and comprehensive, two key criteria for obtaining valid indications of factors affecting the current forest estate market (and detecting possible differences from previous conditions). Furthermore, the analysis of solely forest properties provided more reliable results than the analysis of the full dataset, which is consistent with expectations as including properties with additional, non-forest assets increases risks of confounding results related to the forest valuation.

In summary, our results suggest that the standing volume per hectare and region are the main determinants of a Swedish forest estate's price in the current market, while site quality and forest age (which strongly influence net present value) apparently have little influence. However, detailed studies of a small number of properties may be needed to identify additional influential factors and test the conclusions more rigorously. For example, our use of proportions of the forests exceeding defined ages to test the effect of age distribution on prices may have been too simplistic, and there may be correlations between the proportion of bare forest land and price. Similarly, other age-related factors that have not been considered and/or the proportion of mature forest according to Swedish Forestry Act definitions may influence prices. Thus, using more variables to characterize the age distribution of the estates' forests more thoroughly may provide more robust conclusions about its effect on prices.

\section{Acknowledgements}

We are grateful for constructive help with the regression analysis from Associate Professors Anders Muszta, Dept. of Forest Economics and Sören Holm, Department of Forest Resource Management, SLU, Umeå. We also thank Sees-editing for checking the language.

\section{References}

Andersson. G., Jorner. U., \& Ågren, A. (2007). Regressions-och tidsserieanalys. Lund: Studentlitteratur. (in Swedish)

Aronsson, A., \& Carlén, O. (1997). The Determinants of Forest Land Prices-An Empirical Analysis (Arbetsrapport 242). Umeå: Institutionen för skogsekonomi, SLU.

Faustmann, M. (1849). Calculation of the Value Which Forest Land and Immature Stands Possess for Forestry. Allgemeine Forst- und Jagdzeitung, 15, 441-55

Kennedy, G. A. (2002). Estimating Tract Value Relationships in the North Louisiana Timberland Market. Journal of the Southwestern Economic Review, 26,123-134.

Lantmäteriet (2012). http://www.lantmateriet.se/templates/LMV_Page.aspx?id=3509

Lantmäteriet och Mäklarsamfundet (Land Survey and Real Estate Agency) (2010). Fastighetsvärdering—grundläggande teori och praktisk värdering. LMV-Rapport. Gävle: Lantmäteriverket; Solna: Mäklarsamfundet. (in Swedish)

Lindeborg, T. (1986). Icke-monetära nyttors betydelse för värdet av skogsfastigheter. Stockholm: Tekniska högskolan. (in Swedish)

LRF Konsult (2012). http://lrfkonsult.se/press/Nyheter/Skogsmarkspriser-2011/

Roos, A. (1996). A Hedonic Price Function for Forest Land in Sweden. Canadian Journal of Forest Research, $26,745$.

Scarpa, R, Buongiorno, J., Hseu, J.-S., \& Abt, K. L. (2000). Assessing the Non-Timber Value of Forests: A Revealed-Preference, Hedonic Model. Journal of Forest Economics, 6, 83-107.

Skogsstatistisk årsbok (2013). Skogsstatistisk årsbok (English Title: Swedish Statistical Yearbook of Forestry). Jönköping: Skogsstyrelsen (Swedish Forest Agency).

Snyder, S. A., Kilgore, M. A., Hudson, R., \& Donnay, J. (2007). Determinants of Forest Land Prices in Northern Minnesota: A Hedonic Pricing Approach. Forest Science, 53, 25-36.

Sveriges riksdag (Swedish Parliament) (1990). http://www.riksdagen.se/sv/Dokument-Lagar/Forslag/Motioner/med-anledning-av-prop-199091_GE02Jo142/?text=true 
S. Carlsson et al.

Thaler, R. H. (1988). Anomalies: The Winner’s Curse. Journal of Economic Perspectives, 2, 191.

The Swedish National Forest Inventory.

http://www.slu.se/en/webbtjanster-miljoanalys/forest-statistics/statistiks-from-the-swedish-national-forest-inventory/

Turner, R., Newton, C. M., \& Dennis, D. F. (1991). Economic Relationships between Parcel Characteristics and Price in the Market for Vermont forestland. Forest Science, 37, 1150-1162. 
Scientific Research Publishing (SCIRP) is one of the largest Open Access journal publishers. It is currently publishing more than 200 open access, online, peer-reviewed journals covering a wide range of academic disciplines. SCIRP serves the worldwide academic communities and contributes to the progress and application of science with its publication.

Other selected journals from SCIRP are listed as below. Submit your manuscript to us via either submit@scirp.org or Online Submission Portal.
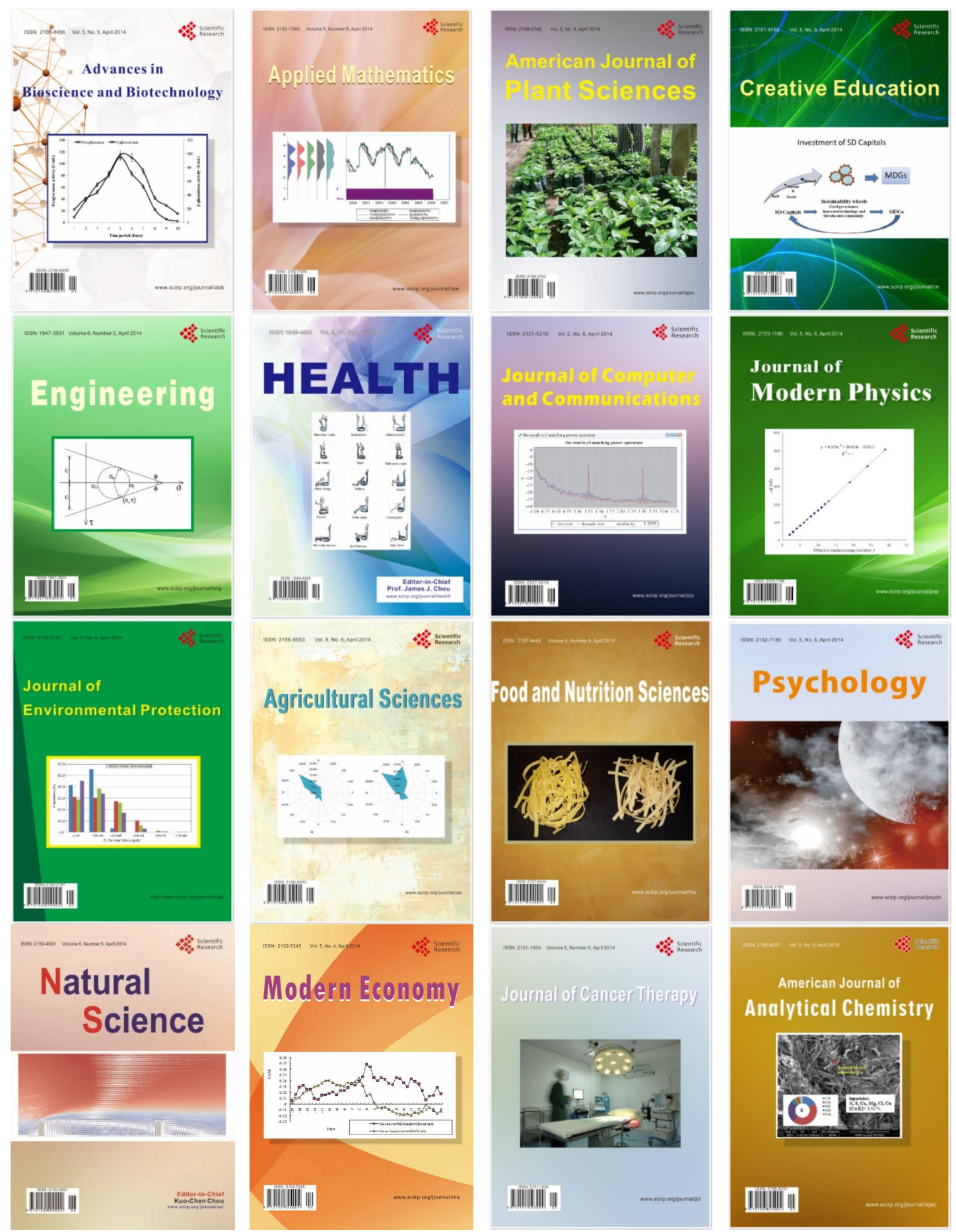